\title{
ON THE NUMBER OF REALIZATIONS OF A HASSE DIAGRAM BY FINITE SETS
}

\author{
ABRAHAM P. HILLMAN
}

R. Dedekind proposed the problem, still unsolved for $n>6$, of finding the order $f(n)$ of the free distributive lattice with $n$ generators. ${ }^{1}$ It is known that $f(n)$ is the number of families $F$ of subsets of a set of $n$ objects such that no subset in $F$ includes any other. ${ }^{2} \mathrm{~A}$ closely related problem is that of finding the number of families of subsets having specified inclusion relations, i.e., a given Hasse diagram. ${ }^{3}$ For families of two subsets, G. N. Raney has solved this problem in terms of a general $n .^{4}$ The present paper contains the solution, by other methods, for families of four or less subsets. In the process, we illustrate both recursive and direct methods of finding the numbers of realizations of Hasse diagrams by subsets of a finite set.

1. Notation. Let $\Sigma$ be a set of $n$ objects. We shall use subset to mean subset of $\Sigma$. By realization of a Hasse diagram will be meant a realization by a family $\alpha_{1}, \cdots, \alpha_{\text {o }}$ of subsets. Let $t$ be the number of inclusion relations among the $\alpha$ 's. Families with different Hasse diagrams may have the same $s$ and $t$, e.g., $s=4$ and $t=5$ for realizations of both

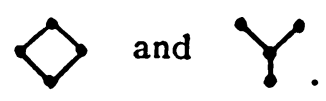

We shall denote by $a_{s t}(n), b_{s t}(n)$, etc. the numbers of realizations of the respective diagrams with a given $s$ and $t$. Dedekind's $f(n)$ may be expressed in the form $\sum_{s=1}^{2^{n}} a_{8 \mathrm{c}}(n)$.

If $g(n)$ is a function defined for non-negative integers, the sum $\sum_{i=0}^{n}{ }_{n} C_{i} g(i)$ will be denoted by $T g(n)$. Clearly $T m^{n}=(m+1)^{n}$. In the natural way this definition is extended to polynomials, with rational coefficients, in $T$, operating on functions $g(n)$.

Received by the editors March 2, 1954.

1 See $[1$, p. 147]. Dedekind gave the order for $n \leqq 4$. R. Church has the order for $n=5$ in [5] and M. Ward the order for $n=6$ in [6].

2 See Theorem 5.14 in [4]. Birkhoff states that this is essenially due to Th. Skolem [2]. See also [3].

${ }^{3}$ A Hasse diagram pictures the order relations in a partially ordered finite set. As applied here, each subset is represented by a vertex and an upward slanting segment is drawn from the vertex for $\alpha$ to that for $\beta$ if $\alpha \subset \beta$ and the family has no $\gamma$ such that $\alpha \subset \gamma \subset \beta$.

- This verbally communicated result inspired the present paper. 


\section{A recursive method.}

Theorem I. Let $D$ be a Hasse diagram with one minimals vertex, $V$. Let $D^{\prime}$ be the diagram obtained by deleting from $D$ the vertex $V$ (and the lines from $V$ ). Let $a(n)$ and $a^{\prime}(n)$ be the numbers of realizations of $D$ and $D^{\prime}$ respectively. Then $a(n)=(T-1) a^{\prime}(n)$ or $T a^{\prime}(n)$, depending on whether $D^{\prime}$ has one or more than one minimal vertex.

Proof. One can choose a subset $\alpha$ of $n-i$ of the $n$ objects in ${ }_{n} C_{i}$ ways. Let $\beta_{1}, \cdots, \beta_{s}$ be any of the $a^{\prime}(i)$ realizations of $D^{\prime}$ by subsets of the remaining $i$ objects. If $D^{\prime}$ has at least two minimal vertices, no $\beta_{j}$ is null and $\alpha, \alpha \cup \beta_{1}, \cdots, \alpha \cup \beta_{s}$ is a realization of $D$. Since each realization of $D$ is obtained uniquely in this way,

$$
a(n)=\sum_{i=0}^{n}{ }_{n} C_{i} a^{\prime}(i)=T a^{\prime}(n),
$$

in this case. If $D^{\prime}$ has only one minimal vertex, it is clear that $a(n)$ is $T a^{\prime}(n)$ minus the number of times, $a^{\prime}(n)$, in which $\alpha$ coincides with an $\alpha \cup \beta_{j}$.

Theorem $I$ is generalized in $\$ 8$ below.

3. The solution for 2 subsets. First let $t=1$. There is just the one diagram

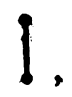

which by Theorem I has $a_{21}(n)=(T-1) 2^{n}=3^{n}-2^{n}$ realizations.

Since the total number of pairs of subsets is $2^{n}\left(2^{n}-1\right) / 2$, we have for $t=0$, i.e., the diagram

$$
a_{20}(n)=\mid 2^{n}\left(2^{n}-1\right) / 2-a_{21}(n)=\left(4^{n}-2 \cdot 3^{n}+2^{n}\right) / 2 .
$$

4. The solution for 3 subsets. The only diagram for $t=3$ is

Using Theorem I, we sẹe that

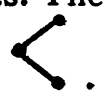

$$
a_{33}(n)=(T-1) a_{21}(n)=4^{n}-2 \cdot 3^{n}+2^{n} \text {. }
$$

By duality the two diagrams,

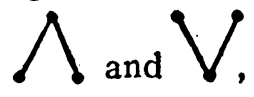

- A minimal vertex is one from which there, are no downward sloping segments. 
for $t=2$ have the same number of realizations. Theorem I shows that this is given by

$$
\mathrm{a}_{32}(n)=T a_{20}(n)=\left(5^{n}-2 \cdot 4^{n}+3^{n}\right) / 2 .
$$

There is just the diagram

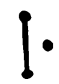

for $t=1$. We obtain the number, $a_{31}(n)$, of realizations by considering the number, $h(n)$, of ordered triples $(\alpha, \beta, \gamma)$ of subsets such that $\alpha$ properly includes $\beta$ and $\gamma$ is different from $\alpha$ and from $\beta$. Clearly $h(n)=\left(2^{n}-2\right) a_{21}(n)$. Also $h(n)$ is the number of realizations of

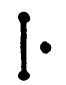

plus three times the number, $a_{33}(n)$, of realizations of

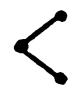

plus two times the number, $2 a_{32}(n)$, of realizations of

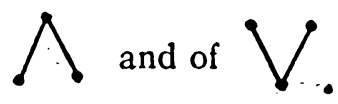

Hence

$a_{31}(n)=\left(2^{n}-2\right) a_{21}(n)-3 a_{33}(n)-4 a_{32}(n)=6^{n}-2 \cdot 5^{n}+2 \cdot 3^{n}-2^{n}$.

Similarly, the number of realizations of the diagram

for $t=0$ is

$$
\begin{aligned}
a_{30}(n) & =\left[\left(2^{n}-2\right) a_{20}-2 a_{32}-2 a_{31}\right] / 3 \\
& =\left(8^{n}-6 \cdot 6^{n}+6 \cdot 5^{n}+3 \cdot 4^{n}-6 \cdot 3^{n}+2 \cdot 2^{n}\right) / 6 .
\end{aligned}
$$

5. The solution for 4 subsets. With the exception of the formulas for $b_{44}(n)$ and $c_{43}(n)$, which are derived in $\$ \S 9$ and 10 respectively, all the material in the following table of solutions for $s=4$ may be found readily by the methods of the previous sections.

\section{Two lemmas.}

Lemma 1. The number of ordered s-tuples $\left(\sigma_{1}, \cdots, \sigma_{s}\right)$ of disjoint subsets is $(s+1)^{n}=T^{s} \cdot 1$.

The essence of the proof is shown by treating the case $s=3$. One 
SOLUTIONS FOR $s=4$

Diagram $\mid$\begin{tabular}{l|l}
$t$ & Number of Realizations \\
\hline & 5
\end{tabular}


may choose $\sigma_{1}$ in ${ }_{n} C_{i}$ ways as a subset with $i$ members. In ${ }_{n-i} C_{j}$ ways, $\sigma_{2}$ may then be chosen as a subset with $j$ members. One chooses $\sigma_{3}$ as any of the $2^{n-i-j}$ subsets of the remaining $n-i-j$ objects. The total number of such triples is thus

$$
\sum_{i=0}^{n}{ }_{n} C_{i} \sum_{j=0}^{n-i}{ }_{n-i} C_{j} \cdot 2^{n-i-j}=\sum_{i=0}^{n} 3^{n-i}{ }_{n} C_{i}=4^{n} .
$$

In a similar fashion one obtains

Lemma 2. The number of ordered s-tuples $\left(\sigma_{1}, \cdots, \sigma_{s}\right)$, such that the $\sigma_{i}$ are disjoint subsets and $\sigma_{1}, \cdots, \sigma_{q}$ are not empty is $T^{s-q}(T-1)^{q} \cdot 1$.

7. The direct method. Let $\alpha_{1}, \cdots, \alpha_{s}$ be a family of subsets. In the ordered $s$-tuple $\left(u_{1}, \cdots, u_{s}\right)$ let each $u_{i}$ be 1 or 0 . Let $\rho_{k}$, where $k=\sum_{i-1}^{s} 2^{i-1} u_{i}$, designate the intersection $\alpha_{1}^{\prime} \cap \alpha_{2}^{\prime} \cap \cdots \cap \alpha_{i}^{\prime}$, in which $\alpha_{i}^{\prime}$ is $\alpha_{i}$ if $u_{i}=1$ and $\alpha_{i}^{\prime}$ is the complement in $\Sigma$ of $\alpha_{i}$ if $u_{i}=0$. It is clear that all families of subsets, for which a given selection of the $\rho_{k}$ are not empty and the remainder are empty, have the same Hasse diagram. Since the $2^{s}$ subsets $\rho_{k}$ are disjoint, it follows from Lemma 2 that the number of realizations of a diagram $D$ is

$$
\frac{1}{m} \sum_{j=0}^{2 s} q_{j}(T-1)^{i \cdot 1}
$$

where $q_{j}$ is the number of divisions, leading to the diagram $D$, of the $\rho_{k}$ into $j$ nonempty ones and $2^{s}-j$ empty ones, and where $m$ is the number of inclusion (and noninclusion) preserving permutations of a family realizing $D$.

8. On "products" of diagrams. The following generalization of Lemma 1 is obtained in the same way as the formula of the previous section.

Lemma 3. Let $\left(D_{1}, \cdots, D_{r}\right)$ be an ordered r-tuple of Hasse diagrams. Let $D_{i}$ have $A_{i}(T) \cdot 1$ realizations. Then $A_{1}(T) \cdots A_{r}(T) \cdot 1$ is the number of ordered $r$-tuples $\left(F_{1}, \cdots, F_{r}\right)$ of families of subsets such that $F_{i}$ is a realization of $D_{i}$ and each subset in $F_{i}$ is disjoint with each subset in $F_{j}$ for $i \neq j$.

Using Lemma 3, Theorem I is easily generalized as follows:

Theorem II. Let $D^{\prime}$ and $D^{\prime \prime}$ be Hasse diagrams. Let $D$ be the diagram formed by placing $D^{\prime}$ below $D^{\prime \prime}$ and drawing segments upward from each maximal vertex of $D^{\prime}$ to each minimal vertex of $D^{\prime \prime}$. Let $A(T) \cdot 1, A^{\prime}(T) \cdot 1$, and $A^{\prime \prime}(T) \cdot 1$ be the numbers of realizations of $D, D^{\prime}$, and $D^{\prime \prime}$ respectively. Then $A(T)=A^{\prime}(T) A^{\prime \prime}(T)$ if either $D^{\prime}$ has more than one maxi- 
mal vertex or $D^{\prime \prime}$ has more than one minimal vertex. If there is only one maximal vertex $V^{\prime}$ of $D^{\prime}$ and only one minimal vertex $V^{\prime \prime}$ of $D^{\prime \prime}$, then $A(T) \cdot 1=A^{\prime}(T) A^{\prime \prime}(T) \cdot 1$ minus the number of realizations of the diagram formed by placing $D^{\prime}$ and $D^{\prime \prime}$ so that $V^{\prime}$ and $V^{\prime \prime}$ coincide.

9. Calculation of $b_{44}$. Applying Theorem II with $D$ chosen as

and both $D^{\prime}$ and $D^{\prime \prime}$ as

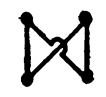

we have

$b_{44}(n)=\left[T(T-1)^{2} / 2\right]^{2} \cdot 1=\left(7^{n}-4 \cdot 6^{n}+6 \cdot 5^{n}-4: 4^{n}+3^{n}\right) / 4$.

10. Calculation of $c_{43}$. Using the notation of $\$ 7$, we find the number of realizations of

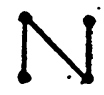

as follows. Examination shows it to be necessary and sufficient for a family $\alpha_{1}, \alpha_{2}, \alpha_{3}, \alpha_{4}$.to have just the inclusion relations $\alpha_{1} \subset \alpha_{2}$, $\alpha_{3} \subset \alpha_{2}$, and $\alpha_{3} \subset \alpha_{4}$ that $\rho_{k}$ be empty for $k=1,4,5,6,7,9,12,13$ and not empty for $k=3,8,14$.

Let $\left(\sigma_{1}, \cdots, \sigma_{7}\right)$ be an ordered septuple of disjoint subsets such that $\sigma_{1}, \sigma_{2}, \sigma_{3}$ are not empty. A family of $\alpha_{i}$ realizing

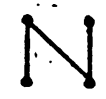

will result if one lets $\rho_{3}, \rho_{8}, \rho_{14}, \rho_{2}, \rho_{10}, \rho_{11}, \rho_{16}$ be $\sigma_{1}, \cdots, \sigma_{7}$ respectively, lets $\rho_{0}$ be the complement in $\Sigma$ of the union of the $\sigma_{j}$, and chooses the remaining $\rho_{k}$ to be empty. Since each realization is obtained exactly once in this way, it follows from Lemma 2 that

$$
c_{43}(n)=T^{4}(T-1)^{3} \cdot 1=8^{n}-3 \cdot 7^{n}+3 \cdot 6^{n}-5^{n} .
$$

11. An observation. In view of the role of the finite difference operator $T-1$ in the above, it is interesting to note that a polynomial $A(T)$, with rational coefficients, is divisible by the $v$ th power of $T-1$ if and only if $a(n)=A(T) \cdot 1$ vanishes for $n=0,1, \cdots, v-1$.

\section{REFERENCES}

1. R. Dedekind, Über Zerlegungen von Zahlen durch ihre grössten gemeinsamen Teiler, Festschrift Techn. Hoch., Braunschweig, 1897, and Gesammelte Werke, vol. 2, pp. 103-148. 
2. Th. Skolem, Om konstitutionen av den identiske kalkuls grupper, Third Scand. Math. Congr., 1913, pp. 149-163.

3. —Über gewisse "Verbände" oder "Lattices," Avh. Norske Vid. Akad. Oslo (1936) pp. 1-16. 1940.

4. G. Birkhoff, Lattice theory, Amer. Math. Soc. Colloquium Publications, vol. 25,

5. R. Church, Numerical analysis of certain free distributive structures, Duke Math. J. vol. 6 (1940) pp. 7.32-734.

6. M. Ward, Bull. Amer. Math. Soc. Abstract 52-5-135.

Columbia University

\section{ON COMPLETE LATTICES AND A PROBLEM OF BIRKHOFF AND FRINK ${ }^{1}$}

\section{K. BALACHANDRAN ${ }^{2}$}

Two decomposition theorems for elements of complete lattices in terms of join prime or completely join prime elements are obtained (Theorems 1 and 2). The latter theorem gives a solution for a problem of Birkhoff and Frink on the relation between completely prime and completely join irreducible ideals. An interesting special case of this solution-for complemented lattices-is also noted (Theorem $3)$.

1. Definitions. An element $a$ of a lattice $L$ is called join irreducible if $a_{1} \cup a_{2}=a \rightarrow a_{1}$ or $a_{2}=a$, and join prime if $a_{1} \cup a_{2} \geqq a \rightarrow a_{1}$ or $a_{2} \geqq a$. Similarly $a$ is called completely join irreducible if (for all existing joins $\left.\bigcup_{i} a_{i}\right) \bigcup_{i} a_{i}=a \rightarrow$ some $a_{i}=a$, and completely join prime if $\cup_{i} a_{i} \geqq a$ $\rightarrow$ some $a_{i} \geqq a$.

The definitions of the corresponding dual concepts (indicated in each case by using the prefix "meet" in the place of "join") are quite clear.

A lattice $L$ will be said to be infinitely meet distributive if all (existing) meets $\bigcap_{i} a_{i}$ in it are distributive; the meet $\bigcap_{i} a_{i}$ is said to

Received by the editors February 26, 1953 and, in revised form, August 15, 1954.

1 The arrangement of the material in the present version follows the suggestions of the referee to whom the author's thanks are due. His thanks are also due to Dr. V.S. Krishnan with whom he has had useful consultations while preparing the manuscript.

2 Government of India Senior Research Scholar. 